How to reference this article

Kenda, J. (2019). Colpa dell'interferenza: analisi contrastiva di errori negli scritti di studenti universitari slovenofoni di italiano. Italica Wratislaviensia, 10(1), 77-109.

DOI: http://dx.doi.org/10.15804/IW.2019.10.1.4

Jana Kenda

Univerza v Ljubljani

jana.kenda@ff.uni-lj.si, ORCID: 0000-0002-6630-6120

\title{
COLPA DELL'INTERFERENZA: ANALISI CONTRASTIVA DI ERRORI NEGLI SCRITTI DI STUDENTI UNIVERSITARI SLOVENOFONI DI ITALIANO
}

\author{
THE BLAME IS ON INTERFERENCE: \\ A CONTRASTIVE ANALYSIS OF ERRORS \\ IN THE WRITING OF SLOVENE-SPEAKING \\ UNIVERSITY STUDENTS OF ITALIAN
}

\begin{abstract}
This contribution aims to address and show the crucial points of mother-tongue interference in the writing of Slovene-speaking learners of Italian. The issue is presented on the basis of a comparison between the language rules in Slovene and Italian in relation to the difficulties encountered in concrete contexts. The goal of this article is to identify the hypotheses underlying learners' language choices. The article illustrates the mistakes made in the choice of verbal paradigms (consecutio temporum; doubts regarding choosing between the perfect and the imperfect and the explicit and implicit forms; some passive constructs; and the subjunctive) as well as the difficulties in using articles and certain adjectives and pronouns (possessive, demonstrative, and relative ones). The work also contains documented and commented examples of errors in the use of the comma and cases of interference that can be attributed to "false friends" (errors of spelling and of a morphological-syntactic, derivational, and lexical nature). The results of this analysis confirm the need for a conscious integration of direct teaching, noticing linguistic mechanisms, and a focus on form in learning processes in order to regulate the input, facilitate understanding, and stimulate natural processes of acquisition.
\end{abstract}

Keywords: Slovene-speaking learners of Italian, contrastive analysis, mother-tongue interference, summary, error analysis 


\section{INTRODUZIONE}

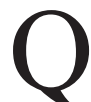
uesto contributo affronta alcuni tra gli errori più ricorrenti e più difficilmente eradicabili negli scritti degli apprendenti slovenofoni di italiano L2. La questione sarà presentata sulla base del confronto tra le norme linguistiche in italiano e sloveno in relazione alle difficoltà riscontrate in contesti concreti e attraverso l'illustrazione degli esempi più significativi. L'obiettivo, data l'ampiezza del problema appena enucleato, non può evidentemente essere quello di dare una panoramica esaustiva di tutta la casistica, ma piuttosto di identificare, puntando su uno sguardo contrastivo, le ipotesi che stanno alla base delle scelte linguistiche degli apprendenti. La prospettiva contrastiva non è certamente l'unico modo per individuare e con ciò eliminare le insicurezze e gli sbagli negli apprendenti di italiano L2, ma è sicuramente uno dei mezzi imprescindibili del loro sviluppo linguistico, soprattutto a livelli di lingua più alti.

Il contributo è organizzato come segue. Presenteremo brevemente le due nozioni di «norma» linguistica e di «errore», per mettere in rilievo alcuni aspetti importanti per la discussione finale. Successivamente passeremo in rassegna i dati concernenti la scelta del corpus e il profilo degli studenti, ovvero gli autori degli esempi citati. Seguirà l'illustrazione dei risultati dell'analisi basata su una classificazione che distingue gli errori di natura morfologica, sintattica, ortografica e lessicale. Nella discussione finale cercheremo di trarre conclusioni riassuntive indirizzate soprattutto a una possibile revisione dei punti su cui focalizzare l'attenzione nell'affrontare gli argomenti problematici insieme agli apprendenti.

\section{IL CONCETTO DI ERRORE E DI NORMA NELL'OTTICA DELL'APPRENDIMENTO DI L2}

Anche se pare logico e chiaro, almeno a livello intuitivo, che cosa sia un errore, la formulazione del concetto è tutt'altro che univoca. Nell'intento di dare una definizione quanto più concisa e delimitativa, ci affidiamo alle parole di Grandi, che descrive l'errore come «un insieme molto va- 
sto e articolato di fenomeni linguistici, accomunati dall'essere percepiti in qualche modo come devianti rispetto ad una regola o ad una norma» (2015, p. 21). Il riferimento alla mancata riuscita di una realizzazione linguistica ci induce a ritenere che, se esiste una deviazione o una violazione della norma, ci deve essere un modello di varietà corretta con il quale è possibile fare paragoni. Essendo la nostra ricerca circoscritta al campo dell'acquisizione dell'italiano come L2, l'errore va ulteriormente precisato come un elemento linguistico (o una combinazione di elementi) che in un determinato contesto e in determinate condizioni con molta probabilità non verrebbe prodotto da un parlante nativo (cf. Lennon, 1991). L'errore presuppone infatti un parlante che parli una lingua «non sua», cioè una lingua in fase di apprendimento, mentre un parlante nativo non può, per definizione, fare errori in quanto è proprio in base al suo comportamento linguistico che si costruisce il sistema di quella determinata lingua (cf. Cadorna, 1988; Andorno, 2015).

Pertanto il parametro di valutazione è la norma standard di una comunità linguistica di parlanti nativi dove la norma prevede sia regole che definiscono o descrivono le diverse pratiche sociali e le forme di comportamento linguistico ${ }^{1}$ sia regole che comandano o vietano una determinata condotta linguistica ovvero impongono regole da osservare. ${ }^{2}$ E quando si rilevano degli errori, questi possono essere deviazioni sia dall'uso pratico che dalla norma. Nei parlanti nativi si riscontrano solo errori del secondo tipo, mentre nei parlanti di L2 sono di ambo i tipi. Si tratta di errori di apprendimento e acquisizione che Grandi definisce intenzionali, ma inconsapevoli: l'apprendente applica di propria volontà una regola che fa parte della sua competenza e che è frutto di un processo di astrazione a partire da un input. Facendo ciò, incorre in errore perché non si rende conto che questo comportamento linguistico diverge dal comportamento dei parlanti madrelingua di quella determinata comunità, che parte dallo stesso input (2015, p. 22).

1 «Regole regolative» secondo Berruto (2015, p. 45).

2 «Regole costitutive» secondo Berruto (ibidem). 
Nonostante il parametro di valutazione sia la lingua target $^{3}$ che imprescindibilmente costituisce lo stimolo che innesca l'acquisizione, gli studi sull'apprendimento di seconde lingue hanno superato la fase in cui le produzioni degli apprendenti venivano valutate solo in confronto con quelle dei madrelingua, ${ }^{4}$ ma sono piuttosto volti a studiare le produzioni degli apprendenti in quanto tali, nell'intento di individuarne il funzionamento e la regolarità. A contribuire notevolmente alla ricognizione della complessità di influssi interlinguistici e intralinguistici e alla necessità di farli confluire nell'analisi è stata l'identificazione dell'interlingua, ${ }^{5}$ una «via di mezzo» tra la condizione del non conoscere e il conoscere una lingua straniera (cf. James, 1998, p. 3) ovvero le diverse varietà di apprendimento di una determinata lingua sviluppate da discenti che la studiano, ma non l'hanno pienamente acquisita.

3 Facciamo uso in questo contesto del termine lingua target invece di lingua straniera o $L 2$ per il suo carattere teleologico che rimanda al reale sforzo degli apprendenti di imparare una lingua straniera (cf. James, 1998, p. 3).

4 James menziona l'analisi contrastiva (Contrastive Analysis) tra i tre (possibili) punti di confronto adottati per le fasi consecutive di apprendimento delle lingue straniere (vedi schema 1) contestandole di essere spesso disinformativa (l'apporto nell'identificazione degli errori non risulta essere innovativo) e imprecisa (gli studi mostrano che gli errori previsti dall'analisi contrastiva non si materializzano regolarmente nell'interlingua e, viceversa, nell'interlingua si manifestano tanti errori che l'analisi contrastiva non può prevedere) (James, 1998, p. 4). In questo contesto si prenda in considerazione anche l'avvertenza di Andorno su come il solo confronto con la lingua target «può non essere la prospettiva migliore da cui osservare la sistematicità di una varietà di apprendimento, che può o meno esemplarsi sulle regole della varietà target» (Andorno, 2015, p. 163), perché si rischia di incorrere in quello che Bley-Vroman chiama la fallacia comparativa (comparative fallacy), che consiste nell'errore di studiare il carattere sistematico di una lingua confrontandola con un'altra (Bley-Vroman, 1983, p. 6).

5 Il concetto di interlingua (interlanguage) attribuito a Selinker (1972) è stato successivamente interpretato, ampliato e ridefinito da diversi autori. Corder (1971) preferisce chiamarlo con il termine dialetto idiosincrasico (idiosyncratic dialect) alludendo ad altre caratteristiche che questa varietà include (cf. James, 1998, p. 3). 
Schema 1: Punti di confronto per le fasi consecutive di apprendimento delle lingue straniere (James, 1998, p. 3)

L1/

lingua madre (LM)

$$
\begin{array}{ll} 
& \text { IL/ interlingua } \\
\text { confronto tra } & \text { LM:LT (analisi contrastiva) } \\
& \text { IL:LT (analisi dell'errore) } \\
& \text { LM:IL (analisi del transfer) }
\end{array}
$$

Gli studi sull'apprendimento di L2, o più precisamente le discipline che studiano le interlingue o varietà di apprendimento, non si limitano nemmeno a una semplice analisi degli errori ${ }^{6}$ commessi dagli apprendenti, ma sono invece interessate alla sistematicità, ovvero alla presenza di regole, piuttosto che alla deviazione. Infatti, come afferma Berruto:

[...] un errore non è generato dal mancato rispetto di una regola, ma da un'altra regola: chi sbaglia secondo una certa norma è perché applica una regola non prevista da quella norma linguistica. [...] L'errore deriva sempre dal confronto con una norma intrinseca od estrinseca. (Berruto, 2015, p. 48)

Ciò che rappresenta appunto l'obiettivo di queste discipline è osservare e capire il meccanismo che induce l'apprendente a commettere errori sistematici e «regolari» nelle diverse fasi della sua interlingua. Infatti, l'apprendente elabora delle ipotesi sulla scorta di un input ${ }^{7}$ nell'intento di costruire un sistema linguistico logico e efficace. In questo modo si innesca un processo mutevole e progressivo che si materializza in «lingue provvisorie, magari lontane dalla lingua obiettivo e quindi in questo senso 'sbagliate', ma non per questo meno coerenti e sistemati-

${ }^{6}$ James annovera anche l'analisi degli errori (Error Analysis) tra i punti di confronto dei processi di apprendimento delle lingue straniere. L'analisi degli errori descrive innanzitutto l'IL dei discenti e la LT, per poi fare successivamente un confronto tra le due con l'intento di individuare le discrepanze (James, 1998, p. 5).

7 Per quanto riguarda un confronto tra l'acquisizione naturale e quella guidata in termini di efficacia e utilità nelle diverse fasi di apprendimento si rimanda allo studio di Andorno (2012). 
che» (Donati, 2013, p. 294). In questa ottica l'errore va visto come un «indicatore di regolarità» (Grandi, 2015, p. 25), una regolarità diversa da quella prevista dalla lingua target. L'errore diventa così uno strumento indispensabile per l'identificazione delle ipotesi che l'apprendente delinea nel processo di apprendimento.

Nel quadro del contesto in cui sorge questo studio ci preme fare una considerazione conclusiva dei punti qui discussi facendo ricorso ad alcuni assunti soggiacenti alla teoria dell'acquisizione delle abilità. Secondo questa teoria, che riconosce ruoli importanti sia ai meccanismi di insegnamento espliciti (in base a conoscenze consapevoli, intuizioni e ipotesi) che a quelli impliciti (induttivi e associativi), l'apprendimento sarebbe garantito dalla «trasformazione di una prestazione controllata in automatica, attraverso la conversione di conoscenze dichiarative o esplicite in conoscenze procedurali o implicite» (Grassi, 2015, p. 178). Si accerta inoltre che, per generare espressioni linguistiche corrette, innovative e adeguate, con il graduale progresso della competenza dell'apprendente, aumentano la necessità di riferimento ai dettagli formali dell'input e la cosiddetta ipotesi del noticing (Schmidt, 1995), secondo cui ciò che gli apprendenti notano nell'input è ciò che diventa materiale di apprendimento. Infatti, per impadronirsi di qualsiasi componente della lingua straniera, il soggetto deve essere in grado di notare lo «scarto» (gap), ovvero la distanza tra la propria prestazione e la lingua target, o il «buco» (hole), ovvero la carenza dei mezzi linguistici necessari nel proprio bagaglio di conoscenze (Grassi, 2015, p. 179). Schmidt sostiene che la capacità di notare, ovvero la consapevolezza dei meccanismi linguistici, è in questo senso sinonimo di attenzione che, opposta a intenzionalità e spontaneità, costituisce un fattore imprescindibile di qualsiasi tipo di apprendimento $(1995$, p. 1).

\section{I DATI}

3.1. Profilo degli studenti (autori degli esempi citati)

Questo lavoro prende in considerazione i dati pervenuti dall'analisi dei lavori scritti di studenti del III anno di Italianistica presso la Facoltà 
di Lettere e Filosofia (Filozofska fakulteta) dell'Università di Lubiana, Slovenia. Nonostante l'analisi sia stata svolta sui lavori di sette generazioni (2011-2017), il profilo degli studenti è definibile attraverso alcuni parametri ripetitivi: età (tra i 22 e i 24 anni), lingua madre (prevalentemente slovenofoni, ma in ogni generazione ci sono alcuni studenti bilingui di sloveno e croato/serbo/bosniaco o macedone), curriculum universitario (sono tutti studenti di due indirizzi di studio paralleli ${ }^{8}$ ), livello di conoscenza dell'italiano (B1-B2), conoscenza di altre lingue straniere (per la maggioranza degli studenti l'inglese costituisce la prima lingua straniera appresa durante l'iter scolastico; il livello di conoscenza conseguito è $\mathrm{B} 2 / \mathrm{B} 2+$; le altre lingue più frequentemente parlate, ma a volte a livelli di conoscenza inferiori, sono il tedesco, lo spagnolo e il francese). Quello che differenzia gli studenti di Italianistica, però, è il livello di conoscenza della lingua italiana prima dell'entrata in facoltà: alcuni studenti (che sono in minoranza, si tratta di $2-3$ studenti a generazione) sono originari del Litorale sloveno ${ }^{9}$ e sono esposti sia all'acquisizione naturale (anche se limitata a determinati contesti e situazioni) che a quella guidata, mentre gli altri studenti (che costituiscono la maggioranza) provengono da altre zone dove l'italiano è una delle materie a scelta nelle scuole medie e al liceo.

\subsection{Corpus}

\subsubsection{Perché proprio il riassunto}

Saper sintetizzare e riproporre con parole proprie ciò a cui si è assistito o ciò che si è letto è una delle competenze fondamentali sia nella sfera

8 La Facoltà di Lettere e Filosofia dell'Università di Lubiana offre ai propri studenti una vasta gamma di indirizzi di studio, da quelli filologici (1. romanze, slave, germaniche, asiatiche o classiche, linguistica comparata) a quelli umanistici (sociologia, storia, storia dell'arte, filosofia, letteratura comparata, psicologia, pedagogia e andragogia, geografia, archivistica e biblioteconomia) tra cui gli studenti possono liberamente scegliere.

9 In determinati comuni di questa regione (Isola, Pirano, Capodistria) l'italiano è considerato lingua dell' ambiente e fa parte del curriculo obbligatorio nelle scuole in lingua slovena. 
professionale che in quella privata. Nonostante la ricorrenza e la vastità di contesti in cui ci viene chiesto di esercitarla (stendere il verbale di un incontro di lavoro, una conferenza o un laboratorio, fare gli appunti per riepilogare il contenuto di una lezione, fornire la sintesi di un film o una rappresentazione teatrale, riportare in sintesi ciò che è successo in un evento a cui si è partecipato, ecc.), si tratta di una prova esigente e complessa che attiva una combinazione di competenze prima di tutto cognitive e solo successivamente linguistiche (cf. Cacchione, 2011, p. 15). Infatti, la sintesi presuppone innanzitutto la corretta interpretazione/comprensione del testo di partenza (il che comporta spesso, soprattutto per gli studenti stranieri, anche la consultazione di dizionari, manuali, siti internet) grazie alla quale vengono raccolte le informazioni necessarie per la stesura del proprio lavoro di sintesi. Anche la seconda fase di quest'operazione si dimostra alquanto impegnativa in quanto viene messa in atto la capacità di gerarchizzazione dei dati che porta all'eliminazione di quelli che non si reputano necessari per una fedele riproduzione in forma sintetica. Alla fase della scrittura si arriva quindi solo dopo aver percorso un iter dinamico e attivo in cui si è soggetti a varie inferenze linguistiche e extralinguistiche e si è coinvolti prima nella decodificazione del messaggio, poi nella selezione e elaborazione delle informazioni, arrivando alla concretizzazione nella parafrasi e nella riduzione all'essenziale del materiale da riprodurre (cf. Cacchione, 2011, p. 15; Fornasiero \& Tamiozzo Goldman, 2013, p. 93).

Proprio grazie alla sua polivalenza, la prova del riassunto si mostra come una valida e significativa verifica del livello di conoscenza della lingua e di una serie di abilità consolidate (il rispetto delle regole ortografiche, la corretta applicazione della consecutio temporum e delle altre regole morfosintattiche, il corretto uso di articoli e preposizioni, la giusta scelta degli elementi lessicali e dei connettivi ecc.). Come osservano Fornasiero \& Tamiozzo Goldman, nel riassunto non è possibile «barare» (2013, p. 87) perché questo esercizio denuncia in modo infallibile sia la cattiva comprensione che l'incapacità di rigore logico nella selezione dei dati nonché l'inabilità a ricomporre una parafrasi sommaria facendo ricorso a mezzi linguistici adeguati. E infine, nell'intento di ottenere 
lavori del tutto individuali che coinvolgono i soggetti al massimo impegno in un mondo così tecnologicamente avanzato, risulta alquanto difficile trovare ancora un tipo di prova scritta che non dia la possibilità della famigerata ricorrenza al copia-incolla. Di conseguenza, il riassunto di un testo di partenza precedentemente sconosciuto agli studenti risulta essere un'ottima scelta.

\subsubsection{Corpus}

Il corpus è costituito da 111 riassunti ad opera di studenti iscritti al modulo di Composizione in lingua italiana. Durante il corso si è lavorato sul consolidamento delle abilità e delle cognizioni di scrittura indispensabili per l'elaborazione testuale e la composizione nonché sull'acquisizione progressiva delle tecniche di scrittura per la realizzazione di varie tipologie di testo, tra cui il riassunto. I lavori in questione sono stati prodotti a casa, dove gli studenti hanno potuto lavorare secondo il proprio ritmo e consultare diversi sussidi didattici.

Per questa prova sono stati proposti quattro diversi testi di partenza: i) un articolo di cronaca (Se la pubblicità fa leva sull'orgoglio patrio, di G. Cosenza, pubblicato sul Fatto Quotidiano); ii) un'intervista televisiva di Fabio Fazio a Pietro Grasso (allora Procuratore nazionale antimafia) a Che tempo che fa (RAITRE, 12/12/2011); parti di due capitoli di libri di storia: iii) La natura della mafia (tratto da: L'Italia del tempo presente di Paul Ginsborg) e iv) Teoria e prassi del fascismo. La struttura organizzativa del fascismo e i suoi uomini (tratto da: Storia d'Italia dal 1861 al 1997 di Dennis Mack Smith).

\section{ANALISI}

Il presente lavoro si concentra su alcune tipologie di errori che risentono dell'interferenza della lingua madre slovena sul piano morfologico, lessicale, sintattico e ortografico. L'analisi qualitativa degli scritti di sette generazioni di studenti ha comunque presentato anche numerosi esempi di errori testuali e stilistici che però non sono stati inclusi nel presente lavoro in quanto non ne è stata individuata la causa nell'interferenza. 
In seguito saranno passati in rassegna i punti più insidiosi riguardo alle principali categorie grammaticali.

\subsection{Verbo}

Possiamo affermare con certezza che una delle difficoltà maggiori per gli apprendenti slavi di italiano L2 è rappresentata dalla scelta delle forme verbali da usare in italiano e gli apprendenti sloveni non fanno eccezione. Dalla nostra ricerca risulta che gli studenti slovenofoni di italianistica incorrono in errori ricorrenti legati $i$ ) all'applicazione delle norme che regolano la concordanza dei tempi verbali all'interno del periodo o testo (consecutio temporum), ii) ai criteri di scelta delle forme verbali nel quadro della dicotomia tra il perfetto e l'imperfetto, iii) alla percezione dei contesti morfologico-sintattici e semantici che richiedono il modo congiuntivo, iv) alla distinzione dell'uso tra le forme verbali esplicite e quelle implicite e v) alla diatesi passiva. Di seguito sono riportati i casi di insidie più frequenti.

\subsubsection{Consecutio temporum}

Quanto alla consecutio temporum, la maggiore discrepanza tra le due lingue si riscontra nella sfera del passato. In italiano le forme verbali adempiono contemporaneamente due mansioni: indicano la collocazione delle azioni nel passato e la loro posizione (temporale) rispetto al punto di riferimento. In sloveno invece esiste una differenza basilare tra la situazione nelle completive da una parte (casi di discorso indiretto) e la situazione negli altri tipi di frasi dipendenti: nelle completive vengono usate le stesse forme verbali che si usano per il non passato (il preteklik/passato per l'anteriorità, il sedanjik/presente per la contemporaneità e il prihodnjik/futuro per la posteriorità), mentre in alcune altre dipendenti (relative, concessive e causali) il preteklik/passato è l'unica forma verbale ammessa per esprimere tutti e tre i rapporti e in altre (finali) è obbligatorio usare il pogojnik/condizionale. Inoltre, in sloveno le relazioni temporali tra le azioni si deducono dal contesto (tramite e grazie alle diverse espressioni di tempo, lessico, ecc.) e dalle informazioni 
ricavabili dalla conoscenza del mondo (cf. Miklič, 1983, 1992).$^{10}$ I seguenti esempi illustrano le diversità fra le due lingue:

Italiano: Disse che non aveva fame perché aveva pranzato già a mezzogiorno e che avrebbe cenato senza di me.

\section{Sloveno:}

dip. completive CONTEMPORANEITÀ Povedal mi je, da je $\mathbf{e}_{\text {sedanjikpresente }}$ lačen. [Mi disse che aveva fame.]

ANTERIORITÀ $\quad$ Povedal mi je, da je jedel preteklikpassato $_{\text {and }}$ že opoldne.

[Mi disse che aveva mangiato a mezzogiorno.]

POSTERIORITÀ

Povedal mi je, da bo večerjal ${ }_{\text {prihodnjik }}$ brez mene.

[Mi disse che avrebbe cenato senza di me.]

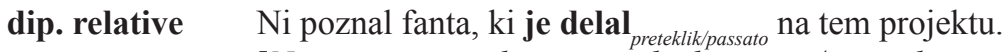
[Non conosceva il ragazzo che lavorava/aveva lavorato/avrebbe lavorato ${ }^{11}$ a quel progetto.]

dip. finali Prišel sem, da bi vam pomagal pogojinikcondizionale $_{\text {. }}$ [Sono venuto per aiutarvi.]

Di conseguenza, il più frequente «tranello» in cui cadono i discenti slovenofoni è quello di non spostare il proprio punto di riferimento al passato e di non fare $\mathrm{i}$ dovuti cambiamenti in accordo con quanto richiesto dalle regole della consecutio temporum italiana, applicando così all'italiano le regole d'uso dei tempi verbali sloveni, come si può vedere nei seguenti esempi tratti dal nostro corpus:

10 «Solo il contesto e il significato dell'insieme orientano nelle frasi slovene l'interpretazione della posizione temporale dell'azione secondaria, mentre nelle corrispondenti italiane, l'interpretazione temporale è suggerita già dal paradigma $[\ldots]$... (Miklič, 1992, p. 200).

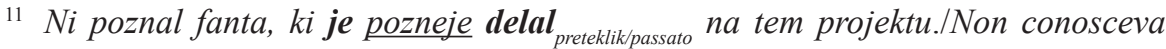
il ragazzo che più tardi avrebbe lavorato a quel progetto. Con l'introduzione di un avverbio di tempo, questa frase relativa esprime anche la posteriorità rispetto a un punto nel passato. 
(1) Falcone fece notare che non cambiò $_{\text {era cambiato }}$ niente in un secolo. ${ }^{12}$

(2) Dietro tutto questo c'è Alberto Fiorello che aveva detto a tuti che avevano dovuto ${ }_{\text {avrebbero dovuto }}$ imporre il tema della mobilità all'interno della campagna elettorale per il sindaco di Roma.

(3) Tutti erano d'accordo che la zona dei Fori Imperiali, che è il parco archeologico più visitato al mondo, si dovrebbe si sarebbe dovuta $_{\text {pedonalizzare. }}$

(4) (...) ha cominciato ad insistere che il problema del traffico a Roma diven$\boldsymbol{t} \boldsymbol{i}_{\text {diventasse }}$ una dei temi della campagna elettorale.

\subsubsection{Scelta tra il perfetto e l'imperfetto}

Per quanto riguarda invece l'insidiosa scelta tra i perfetti (P) e l'IM in italiano, il discente sloveno è incline a trasportare nel contesto italiano i criteri di scelta dello sloveno tra i dovršniki - D/verbi «perfettivi» e i nedovršniki-ND/verbi «imperfettivi», prima di tutto a causa del fatto che in alcune (rare) situazioni è veramente possibile riscontrare una corrispondenza tra le coppie dicotomiche (il perfetto pare corrispondere al $D$, l'imperfetto al $N D)$ :

\begin{tabular}{|c|c|c|c|c|}
\hline Quando & sono uscita & $\begin{array}{l}\text { di casa dopo sette giorni di } \\
\text { influenza, }\end{array}$ & non c'era & $\begin{array}{l}\text { più } \\
\text { la neve. }\end{array}$ \\
\hline \multirow[t]{3}{*}{ Ko } & sem šla ven & po sedmih dneh gripe, snega & ni bilo & več. \\
\hline & $\mathrm{P} \approx \mathrm{D}$ & & $\mathrm{IM} \approx \mathrm{ND}$ & \\
\hline & Feci & $\begin{array}{c}\text { di tutto per arrivare a casa per le } \\
22, \text { ma mia figlia }\end{array}$ & dormiva & \multirow[t]{3}{*}{ già. } \\
\hline \multirow[t]{2}{*}{ Vse } & sem naredil, & da bi se vrnil do $22 \mathrm{~h}$, ampak hči & $\begin{array}{l}\text { je (že) } \\
\text { spala }\end{array}$ & \\
\hline & $\mathrm{P} \approx \mathrm{D}$ & & $\mathrm{IM} \approx \mathrm{ND}$ & \\
\hline
\end{tabular}

12 Nelle citazioni degli esempi illustrativi tratti dal corpus, gli errori che si riferiscono al punto in discussione vengono contrassegnati dalla scrittura in grassetto e corsivo (cambiò) seguita in caratteri piccoli dalla variante corretta. Tutti gli esempi, nonostante la presenza di altri errori, vengono riportati senza alcuna correzione, ovvero in forma originale, così come sono stati prodotti dagli studenti. 
Stando a Miklič (1983), questa è la ragione per cui il discente sloveno tende a generalizzare l'uso dell'IM quando in una situazione analoga slovena avrebbe usato il ND, (come nell'es. 5), e viceversa usa uno dei perfetti (generalmente il PP) quando in sloveno avrebbe usato il D (es. 6): ${ }^{13}$

(5) La prima cosa interessante è che dietro a questo fatto c'è un certo Alberto Fiorillo che ha iniziato tutto questo dicendo che si deve imporre il tema della mobilità all'interno della campagna elettorale. Sopratutto insistevacon i candidati sindaco che hanno lentamente cominciato a partecipare con maggior convinzione. [Vztrajal je /ND/ predvsem pri kandidatih za župana, ki so počasi začeli sodelovati z večjo zavzetostjo.]

(6) Era desiderata l'approvazione straniera per commuovere il popolo italiano, ma anche per fare buona impressione nell'estero. Per questo 1. hanno adattato $_{\text {adattavano }}$ le informazioni nei giornali stranieri e 2. hanno esiliato $_{\text {esiliavano }}$ tutti i giornalisti che 3 . sono stati $_{\text {erano }}$ in contro il fascismo. E ogni giorno 4. ha esaminato $_{\text {esaminava }}$ i giornali esteri per vedere se 5. stanno $_{\text {stavano }}$ scrivendo a suo favore. [Zato so prilagajali informacije $v$ tujih časopisih in izgnali vse novinarje, ki so bili proti fašizmu. In vsak dan je preučil/pregledal tuje časopise, da bi preveril, ali pišejo njemu $v$ prid.]

(Lo studente fallisce a contrassegnare tramite l'imperfetto dei verbi adattare, esiliare e esaminare la cornice di una descrizione di sfondo del contesto storico in cui sono ambientati i fatti. La ragione del mancato uso dell'imperfetto del verbo essere nel caso 3. e stare nel caso 5. è invece da attribuire nuovamente al transfer negativo dallo sloveno per quanto riguarda la concordanza dei tempi al passato).

\subsubsection{Il congiuntivo}

Il modo congiuntivo non esiste nel sistema verbale della lingua slovena. Nell'insegnamento della lingua italiana come L2 si insiste pertanto nella descrizione del suo uso in base $i$ ) alla sua facoltà di esprimere azioni non reali, possibili, desiderate, non desiderate, temute, ecc.; ii) alla sua opposizione con le forme dell'indicativo; iii) ai connettivi e $i v$ ) ai costrutti

13 Per agevolare la comprensione delle ragioni che hanno portato agli errori riscontrati, si è scelto, in alcuni casi, di specificare tra le parentesi quadre i costrutti sloveni sottostanti agli esempi citati. 
sintattici in cui rappresenta l'unica possibilità di scelta. Nonostante l'insistenza su questi aspetti e un intenso lavoro pratico su tutti i tipi di possibili scenari, i due errori che comunemente si manifestano sono quello dell'ipercorrettismo, ovvero dell'impiego del congiuntivo laddove invece si preferisce un altro modo (l'indicativo, per la maggior parte degli esempi) (ess. 7-9 e la seconda forma verbale dell'es. 13: Propone che l'Italia abbia...), e il problema della consecutio temporum nell'ambito della quale gli studenti spesso si rendono conto della necessità di impiegare il congiuntivo, ma sbagliano la forma verbale per esprimere i giusti rapporti temporali rispetto al verbo della frase principale (ess. 10-11):

(7) La pubblicità di Fiat è piena di contraddizioni, ed anche se il suo slogan sia $_{\mathrm{e}}$ «le cose che costruiamo ci rendono ciò che siamo»...

(in questo esempio lo studente riconosce la dipendente concessiva come una delle strutture in cui si usa il congiuntivo, ma non tiene in considerazione che il connettivo anche se viene seguito dall'indicativo)

(8) Compra una cosa non sapendo che si tratti $i_{\text {si tratta }}$ di una impresa illegale e che dennaro $\boldsymbol{s i a}_{\mathrm{e}}$ sporco.

(a causa dell'espressione non sapendo lo studente fraintende il significato e lo interpreta come una situazione di incertezza)

(9) (...) si può dire che chi $\boldsymbol{a b b i a}_{\text {ha }}$ il denaro liquido ha più potere. (la necessità dell'uso del congiuntivo in costrutti con espressioni impersonali viene spesso ipergeneralizzata)

(10) La prima è che non c'è la volontà che qualcuno trattasse tratti $_{\text {questo ar- }}$ gomento.

(11) Si ha bisogno di soltanto una persona ostinata perché il prossimo sindaco di Roma cambiasse $\boldsymbol{e}_{\text {cambi }}$ il destino del Colosseo.

Un frequente caso di mancato uso del congiuntivo si riscontra nei casi di alcune dipendenti (condizionali, finali, oggettive ecc.), in cui la lingua slovena si serve del pogojnik/condizionale. Si osservino alcuni esempi:

- Če bi imel to knjigo, bi ti jo posodil./Se avessi questo libro, te lo presterei.

- Opombe so dodali zato, da bi bila knjiga bolj zanimiva./Hanno aggiunto

dei commenti perché il libro fosse più interessante.

- Bojimo se, da ne bi prišel./Temiamo che possa non venire. 
Seguono ora gli esempi tratti dal nostro corpus in cui è evidente il transfer negativo dallo sloveno:

(12) (...) desiderava fare qualcosa perché il problema della pedonalizzazione nella zona di Fori Imperiali a Roma si sarebbe finalmente risolto . [Želel je nekaj narediti zato, da bi se problem zapiranja prometa $z$ vozili zaradi pešcev v coni Rimskega Foruma končno rešil.]

(13) Propone che l'Italia $\boldsymbol{a b b i a}_{\text {ha }}$ bisogno di una sola persona ostinata che potrebbe obbligare ${ }_{\text {obblighi }}$ il futuro sindaco di Roma per inizare a preoccuparsi per il Colosseo. [Meni, da Italija potrebuje eno samo vztrajno osebo, ki bi prisilila župana, da se začne ukvarjati s Kolosejem.]

\subsubsection{Forme verbali implicite}

Per quanto riguarda le forme verbali implicite, da una parte tra lo sloveno e l'italiano esiste una corrispondenza formale, dall'altra invece le due lingue differiscono notevolmente rispetto all'uso di tali forme in funzione di predicato nelle subordinate.

Accanto alle due forme del modo infinito, nedoločnik/infinito (govoriti/parlare; teči/correre) e namenilnik/supino (grem spat/vado a dormire;), lo sloveno distingue fra: $i$ ) deležje/participio invariabile in funzione

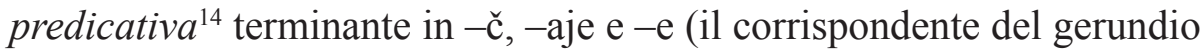
semplice italiano: rekoč/dicendo; prepevaje/cantando; molče/tacendo); ii) deležje terminante in -ši (il corrispondente del gerundio composto italiano: videvši/avendo visto); iii) deležnik/participio, di cui sono usate come forme verbali: opisni deležnik/participio descrittivo, terminante in -1 (začel/iniziato) usato per formare la forma attiva dei tempi composti (je začel/ha iniziato, bi zgorel/brucerebbe) e trpni deležnik/participio passivo, terminante in $-\mathrm{n}$ e - $\mathrm{t}$ (popravljen/riparato, prijet/preso) per formare la forma passiva (bo popravljen /sarà riparato) (Toporišič, 2004, pp. 336-342).

Il punto di distacco tra le due lingue si rileva quindi nell'impiego di queste forme verbali in frasi dipendenti. Mentre in italiano se ne re-

14 «nesklonljivi deležnik v funkciji povedka» - denominazione ripresa da Slovar slovenskega knjižnega jezika (Ahlin et al., 1994, p. 124). 
gistra un'alta frequenza d'uso, ${ }^{15}$ tutti i più recenti studi svolti sulle forme verbali implicite in sloveno avvertono un certo disagio dei parlanti nativi a usare le forme verbali implicite in questa funzione in quanto le percepiscono come obsolete e riservate a registri letterari e formali (Mikolič Južnič, 2017, p. 33). ${ }^{16}$ Lo testimoniano anche i risultati dello studio comparativo di Mikolič Južnič (2017) sulla traduzione dall'italiano in sloveno, che rilevano il ricorso a strutture alternative (tra cui soprattutto la nominalizzazione e le forme verbali esplicite) da parte dei traduttori sloveni. Inoltre, molte delle forme verbali implicite in sloveno vengono ormai usate solo come avverbi (molče/silenziosamente), preposizioni (glede, glede na/riguardo a, quanto a) e aggettivi (goreč skedenj/fienile bruciante, in fiamme, utrujen/stanco) (Toporišič, 2004, pp. 340-341).

In accordo con queste costatazioni è possibile intravedere l'interferenza dello sloveno anche nei lavori analizzati in questo studio.

(14) Vedendo $\boldsymbol{V}_{\text {Visto }}$ il successo che ha avuto quest'idea, c'è maggiore speranza che la situazione cambierà. [Glede na uspeh, ki ga je imela ta ideja, je več upanja, da se bo situacija spremenila.] (Lo studente probabilmente riconosce l'espressione glede come deležje del verbo gledati. Per

${ }_{15} \mathrm{Ci}$ preme aggiungere le dovute osservazioni su alcune differenze nella frequenza d'uso delle singole forme in italiano: $i$ ) il gerundio semplice ha un uso più generale di quello composto, riscontrabile soprattutto in registri alti, letterari o burocratici (Lonzi, 1988, p. 571); ii) ci sono notevoli restrizioni riguardo al tipo di participio passato che può essere usato nelle frasi participiali (è possibile con il part. passato dei v. telici, nondurativi, transitivi, causativi ecc.) (Bertucelli Papi, 2001, p. 593); iii) l'uso verbale del participio presente è riservato ai registri alti, ricercati e burocratici (Benincà \& Cinque, 2001, p. 604).

${ }_{16}$ Da quanto risulta dallo studio di Jesenšek (1998, p. 15), in sloveno si assiste a un'evidente riduzione dell'uso delle forme verbali implicite nelle secondarie, soprattutto di deležnik e deležje in -č e -ši, a cavallo tra il XIX e il XX secolo, soprattutto sotto l'influsso degli scrittori (in particolare Ivan Cankar) e linguisti (tra cui Anton Breznik, che nella sua opera Življenje besed del 1967 critica apertamente questa usanza considerandola un'interferenza dal francese e preferendo categoricamente le forme verbali esplicite). Come afferma Jesenšek, dato che i suggerimenti di Breznik sono stati largamente condivisi, queste forme verbali sono quasi completamente scomparse e il loro uso, riservato solo ad alcuni verbi, è limitato a contesti stilisticamente marcati (ibidem). 
questo sceglie il corrispondente gerundio in italiano, non tenendo in considerazione che avrebbe dovuto esprimere il rapporto di anteriorità rispetto alla frase principale.)

(15) Una delle maniere per ottenere la liquidità è investendo investire $_{\text {nei su- }}$ permercati, perciò, anche $\boldsymbol{s e}_{\text {pur }}$ non sapendo, tutti possiamo essere in contatto con il riciclaggio del denaro sporco. [Eden izmed načinov za doseganje likvidnosti je naložba v veleblagovnice, saj vsi lahko pridemo $v$ stik z umazanim denarjem, ne da bi se tega zavedali.]

(Lo sloveno non conosce l'uso dell'infinito in funzione di complemento predicativo; di solito si ricorre alla nominalizzazione, come naložba in questo caso. La ragione della scelta sbagliata del gerundio sta probabilmente nel transfer dall'inglese. Infatti, in inglese il gerundio ha anche un valore sostantivale, analogamente al valore dell'infinito in italiano. In questo esempio si registra anche l'uso sbagliato della congiunzione anche se usata al posto di pur).

(16) Ha ingannato alla gente con sua laboriosità come ad esempio che ha Iasciato $_{\text {lasciando }}$ la luce accesa tutta la notte per fargli credere che stava ancora lavorando. [Zavajal je ljudi s svojo delavnostjo tako, da je celo noč puščal prižgano luč, da bi verjeli, da še dela.]

(In questo esempio si tratta del tipico transfer negativo del costrutto esplicito sloveno laddove in italiano andrebbe usato il costrutto implicito, ovvero il gerundio, data l'identità dei soggetti delle frasi principale e subordinata.)

(17) Tutti i politici sono d'accordo di farne che bisogna fare $_{\text {qualcosa. [Vsi politiki }}$ se strinjajo, da je treba nekaj narediti.]

(L'errore commesso in questo esempio è quello di generalizzare la situazione in cui viene rilevato il rapporto di contemporaneità o posteriorità, trascurando però la non identità dei soggetti delle frasi principale e subordinata).

\subsubsection{Diatesi passiva}

La diatesi passiva in sloveno esiste, le ragioni del suo uso sono identiche a quelle nel contesto italiano, ma la sua frequenza d'uso è molto più limitata che in italiano. Logar et al. ricordano che nel passato era addirittura sconsigliata dalle grammatiche (2016, p. 237). I linguisti sloveni si limitano a definirla come una mera trasformazione della forma attiva che in sloveno è più diffusa essendo la forma primaria del verbo (cf. Toporišič, 2004, p. 29; Logar et al., 2016, pp. 237-238). Solitamente poco usata nei testi narrativi e divulgativi, appare più spesso nei lin- 
guaggi settoriali (cf. Toporišič, 2004, p. 297) come garanzia di neutralità e per realizzare la deagentivizzazione. I suddetti parametri giustificano la scarsa familiarità con questa diatesi da parte degli apprendenti sloveni e le difficoltà riscontrate nell'acquisizione delle differenze formali e d'uso tra i due sistemi linguistici.

Uno dei principali punti problematici è dovuto all'incapacità degli apprendenti di riconoscere il valore passato del verbo, ragion per cui copiano formalmente il costrutto sloveno (il verbo ausiliare è alla forma del presente, seguito dal participio passato, invece del passato prossimo dell'ausiliare, a sua volta seguito dal participio passato):

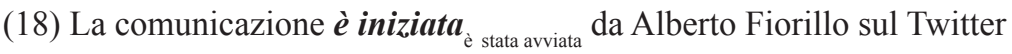

Segue un esempio in cui si riscontra il transfer negativo della transitività del verbo corrispondente: infatti, in sloveno il verbo chiedere viene seguito dall'accusativo indicante la persona a cui si chiede (vprašati nekoga), di conseguenza lo studente traduce liberamente il costrutto passivo: «Vsi tisti, ki so bili vprašani...»:

(19) Tutti quelli che sono stati chiesti $i_{\text {Tutti coloro a cui è stato chiesto }}$ che cosa pensassero dell'idea della pedonalizzazione hanno risposto di sì. [Vsi tisti, $k i$ so bili vprašani, kaj si mislijo o omejitvi prometa z avtomobili v korist pešcev, so odgovorili pozitivno.]

Il seguente caso illustra invece un caso di transfer negativo legato alla questione della forma: lo studente usa erroneamente la forma passiva del passato prossimo (sono stati preparati) a causa dell'influsso dello sloveno che vede concorrere due forme verbali composte da tre elementi (so bili pripravljeni/erano disposti e so bili prisiljeni/sono stati costretti), di cui la prima rappresenta un predicato nominale al passato (v. biti/ essere seguito dal participio passato in funzione aggettivale pripravljen/ disposto) e la seconda la forma passiva del passato del verbo prisiliti: ${ }^{17}$

${ }_{17}$ Il verbo prisiliti è stato scelto qui solo come un esempio per illustrare la forma del passato al passivo di un verbo sloveno. 
(20) Ha insistito in particolare con i candidati sindaco che sono stati preparati $_{\text {erano disposti }}$ a partecipare.

Un ultimo, ma frequente tipo di errore riguardo alla diatesi passiva si riferisce all'uso del si passivante che ricalca la formazione del passivo in sloveno con il morfema se. In sloveno questo costrutto si forma tramite la trasformazione dell'oggetto diretto della frase attiva dall'accusativo al nominativo, seguito dalla $3^{\text {a }}$ persona singolare o plurale del verbo e dal morfema se: Išče se Urša Plut (nominativo) $\approx$ Si cerca Urša Plut. Esiste però un'altra variante di questo costrutto in cui, nel passaggio al costrutto con il morfema se, il caso accusativo dell'oggetto diretto della frase attiva non viene trasformato in nominativo, ma rimane accusativo: Išče se Uršo Plut (accusativo) $\approx$ Si cerca Urša Plut (la forma verbale è sempre alla $3^{\mathrm{a}}$ persona singolare, perché non c'è un elemento al nominativo con il quale accordarla). Questo va interpretato come un costrutto impersonale (equivalente al si impersonale in italiano). Si veda un'ulteriore illustrazione della differenza fra i due:

f. passiva:

f. impersonale:
Iščejo se darovalci krvi (nominativo, il verbo è alla forma plurale)

$\approx$ Si cercano i donatori di sangue.

Išce se darovalce krvi (accusativo, il verbo è alla forma singolare)

$\approx$ Si cercano i donatori di sangue.

Nella produzione in italiano, l'errore degli apprendenti sloveni sta nel mancato accordo del verbo con il soggetto della frase (il costrutto non viene percepito come passivo bensì come impersonale):

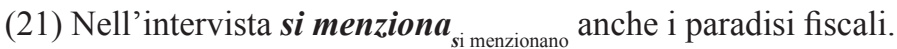

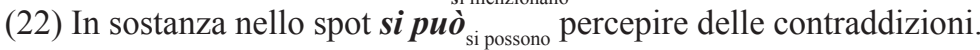

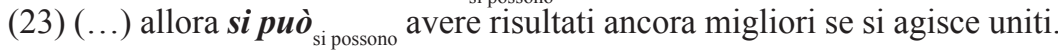




\subsection{ARTICOLO}

Riuscire a comprendere e poi interiorizzare le caratteristiche e gli innumerevoli contesti nella lingua italiana in cui bisogna scegliere fra i diversi tipi di articoli e l'articolo zero, è una delle imprese più ardue per qualsiasi apprendente straniero, soprattutto se la sua lingua madre non conosce la categoria dell'articolo. È necessario in primo luogo capire quando l'articolo realizza la funzione di quantificatore e quando di determinatore e in secondo luogo quanto sulla scelta dell'articolo influisce la notorietà del SN e quanto la sua specificità o la non-specificità: infatti, alle due forme dell'articolo, determinativo e indeterminativo, ${ }^{18}$ dal punto di vista semantico corrispondono tre tipi di sintagmi i cui referenti, al momento dell'enunciazione, possono essere percepiti come noti i) al parlante e (a suo giudizio) all'ascoltatore ( $S N$ determinato: la porta/le porte); ii) al parlante ma (a suo giudizio) non all'ascoltatore ( $S N$ indeterminato specifico: una porta/delle porte) e ii) né al parlante né (a suo giudizio) all'ascoltatore (SN indeterminato non-specifico: una porta/delle, $\varnothing^{\text {art }}$ porte $^{19}$ ) (Renzi 1988: 365). In aggiunta a questi criteri di scelta, i parlanti devono tenere in considerazione anche la distribuzione degli elementi nella frase e la tipologia del N (numerabile/di massa, proprio/ comune, ecc.), della frase (positiva/negativa) o delle formazioni idiomatiche di cui il SN fa parte (cf. Renzi, 1988). Ma fra tutte queste situazioni sembra primeggiare la questione che riguarda la percezione della determinatezza che si manifesta sia nella mancata espressione dell'articolo

18 Come sostiene Renzi, l'articolo partitivo è una variante del secondo (1988, pp. 363-364).

19 Il SN indeterminato specifico e quello non-specifico si distinguono solo, anche se non obbligatoriamente, al plurale: l'assenza del determinante, ovvero l'articolo zero $\left(\varnothing^{\mathrm{art}}\right)$ (per es. in: Ciò che rende importante la Fondazione è che può spalancare $\emptyset^{\text {art }}$ porte, lanciare $\emptyset^{\text {art }}$ carriere.), è possibile solo in caso di $S N$ indeterminato nonspecifico. Nel caso di $S N$ indeterminato specifico, l'articolo zero non è accettabile (*Dobbiamo ristrutturare porte che si trovano al I piano del castello. Accettabile: delle porte) (cf. Renzi, 1988, p. 363). 
(ess. 24-30) sia nell'ipercorrettezza, ovvero nell'uso dell'articolo laddove andrebbe omesso (ess. 31-35):

(24) $\emptyset^{\text {art }}$ Colosseo $_{\text {II Colosseo }}$ è diventato così una delle più grandi rotatorie del mondo... ${ }^{20}$

(25) Se tutti $\mathscr{\emptyset}^{a r t}$ italiani ${ }_{\text {gli italiani }}$ fossero più interessati alla vita politica...

(26) Tutto dipende da $\mathscr{\emptyset}^{\text {art }}$ denaro $_{\text {dal denaro }}$ con cui si può comprare...

(27) [...] in $\emptyset^{\text {art }}$ suo libro ${ }_{\text {nel suo libro }}$ Denaro sporco...

(28) Grasso presenta $\emptyset^{\text {art }}$ coinvolgimento ${ }_{\text {il coinvolgimento }}$ delle mafie nel riciclaggio. $\varnothing^{\text {art }}$ Procuratore ${ }_{\text {II Procuratore }}$ procede con l'esposizione delle tre fasi del riciclaggio...

(29) L'intervista si conclude con $\emptyset^{\text {art }}$ dibattito $_{\text {con il dibattito }}$ sull'individualismo.

(30) Un grande problema sono anche $\mathscr{O}^{a r t}$ paradisi fiscali ${ }_{\text {paradisi fiscali. }}$

(31) [...] l'aiuto segreto degli alcuni ${ }_{\text {di alcuni }}$ suoi componenti...

(32) $[\ldots]$ persino si può fare $l \boldsymbol{a}$ carriera fare carriera $\ldots$

(33) Il tema era la mobilità della Roma $_{\text {di Roma. }}$

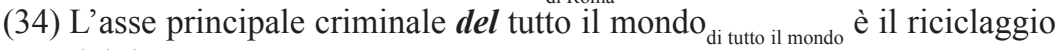
del denaro sporco.

(35) Questo si può chiamare anche $l^{\prime}$ omertà ${ }_{\text {omertà }} \ldots$

La difficoltà di percepire la tipologia semantica del SN ( $S N$ determinato; SN indeterminato specifico o $S N$ indeterminato non-specifico, cf. Renzi, 1988, p. 364) sta alla base delle sostituzioni dell'articolo determinativo con quello indeterminativo e viceversa:

(36) Ciò può essere $\boldsymbol{l} \boldsymbol{a}_{\text {una }}$ conseguenza della crisi economica.

(37) $\boldsymbol{U n} \boldsymbol{a}_{\mathrm{La}}$ morale della favola è che una sola ostinatissima persona è sufficiente...

(38) Si potrebbe dire che essa è $\boldsymbol{u} \boldsymbol{n}_{1}$ 'amica migliore del denaro sommerso.

(39) Il suo scopo era di dare $\boldsymbol{u} \boldsymbol{n}_{1}$ 'impressione di uomo savio.

L'insicurezza nell'accezione dell'indeterminatezza di un SN si nota nel frequente uso dell'articolo partitivo come sostituto plurale dell'art. indeterminativo (negli esempi 40-43 sarebbe più opportuna l'omissione dell'articolo):

(40) (...) a livello nazionale procurava dei voti politici

${ }^{20}$ In questi esempi Ø ${ }^{\text {art }}$ segna l'assenza dell'articolo. 
(41) (...) loro facevano delle alleanze e pure delle competizioni

(42) Fiorillo sfrutta Twitter che non permette dei dibattiti lunghi ma solo delle risposte brevi senza argomentazione.

(43) Si sono sviluppati numerosi paradisi fiscali in tutto il mondo dove delle enormi somme di denaro vengono riciclate.

\subsection{Aggettivi e pronomi}

Le più soventi fonti di errori per quanto riguarda l'uso degli aggettivi e dei pronomi possono essere circoscritte a determinate situazioni in cui vengono usate le categorie dei possessivi, dimostrativi e relativi. In diversi soggetti la ripetitività degli errori ne testimonia la sistematicità.

Tra i possessivi una sola forma crea costanti dubbi (svoj/proprio), probabilmente a causa della polifunzionalità del possessivo svoj in sloveno, che può essere abbinabile con qualsiasi soggetto:

(45) (...) tutti i candidati, chiesti per esprimere la $\boldsymbol{s u a}_{\text {loro }}$ opinione, hanno risposto ugualmente: sì.

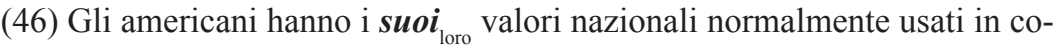
municazione politica.

Non riscontrabile nella lingua slovena è un caso particolare di impiego obbligatorio del dimostrativo quello che, privato del suo valore deittico, sostituisce il nome del predicato (ricavabile a sua volta tramite anafora) davanti a un modificatore introdotto da un sintagma preposizionale o da una frase relativa (Vanelli, 1979, p. 187). Pertanto negli scritti degli apprendenti sloveni si riscontra spesso l'omissione del dimostrativo:

(47) Il servizio più usato era $\boldsymbol{\emptyset}_{\text {quello }}$ di offrire protezione.

(48) Una soluzione possibile sarebbe anche $\mathscr{Ø}_{\text {quella }}$ d'intensificare il controllo sulla provenienza del denaro.

Lo sloveno prevede l'uso del dimostrativo introduttivo to per indicare un essere o oggetto non ancora identificato e per attrarre l'attenzione su di esso. In questo contesto to, che è la forma neutra del dimostrativo ta/questo, rimane sempre invariabile malgrado il genere e numero del sostantivo a cui si riferisce (per es.: To je Tina/Questa è Tina; To sta Tina 
in Marko/Questi sono Tina e Marko), a differenza dell'italiano, che in situazioni analoghe esige l'accordo. Gli apprendenti sloveni applicano erroneamente questa norma e mantengono la forma neutra del pronome dimostrativo introduttivo:

(49) Questo $_{\text {Questa }}$ è la maggiore questione perché lo Stato si occupa solo delle persone. [To je največja težava, ker se država ukvarja samo z osebami.]

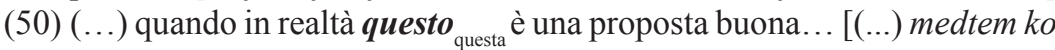
je to pravzaprav dober predlog...]

Un ultimo caso di transfer negativo dallo sloveno in italiano nel quadro delle categorie aggettivali e pronominali si riscontra nell'uso dei pronomi relativi. La difficoltà nasce nel momento in cui, causa la brevità e la paragonabile frequenza d'uso, gli apprendenti sloveni sembrano uguagliare in tutto l'italiano che allo sloveno ki. Quest'ultimo, però, è particolare anche tra le forme contigue in sloveno in quanto nel dativo e accusativo viene inevitabilmente completato dalle forme del pronome personale atono. Nella produzione in italiano degli apprendenti sloveni si riscontra pertanto l'inserimento inappropriato dei clitici accanto a che quando quest'ultimo svolge la funzione di complemento oggetto:

(51) (...) tutti sono d'accordo con questa idea, che $l$ 'ha esposta ${ }_{\text {che ha esposto }}$ il sindaco Petroselli nell'81. [...vsi se strinjajo z idejo, ki jo je predstavil župan Petroselli 1981. leta.]

(52) La struttura della mafia non era esattamente quella che $l$ 'ha descritta ${ }_{\text {che }}$ Buscetta.

(53) (...) a subirne le conseguenze sono i lavoratori che non hanno i diritti che dovrebbero averli $i_{\text {che dovrebbero avere }}$ [...trpijo za posledicam delavci, $k i$ nimajo pravic, ki bi jih morali imeti.]

\section{ORTOGRAFIA}

Uno degli errori più tipici e più ricorrenti nelle produzioni scritte degli slovenofoni, anche a livelli avanzati di conoscenza della lingua italiana, è riservato all'uso della virgola, più precisamente all'aggiunta della virgola laddove di norma non va usata nel contesto della lingua italiana. Si tratta di un esempio tipico di interferenza grammaticale secondo 
Weinreich (1974, p. 45), ovvero dell'applicazione di una regola grammaticale della L1 nella L2. Nel caso concreto, la regola slovena impone in ogni contesto l'uso della virgola per dividere la frase principale da quella subordinata, indifferentemente dalla loro distribuzione e ordine nel periodo (frase dipendente prima o dopo la principale) (Slovenski pravopis, ZRC SAZU, 2001, p. 38). Quello che risulta interessante è che si può parlare di fossilizzazione solo parziale di questa regola in quanto l'errore viene sistematicamente commesso prevalentemente davanti alle dipendenti completive introdotte dalla congiunzione che ( $d a$ in sloveno) e davanti ai pronomi relativi ( $k$ i, kateri in sloveno). ${ }^{21}$

(54) Falcone come palermitano afferma, ç̣ẹ tutti gli abitanti rispettavano l'omertà della mafia.

(55) Sorprende il fatto, che dietro a tutto questo non ci stanno i giornalisti, ma un certo Alberto Fiorillo.

(56) Il capitale sporco e criminalità si annidano, dọyẹ c'è una vulnerabilità democratica...

(57) Interessante è, chẹ il dibattito virtuale è stato organizzato solamente da Alberto Fiorillo.

Tra gli altri errori ortografici più frequenti si annoverano inoltre:

- l'uso della lettera maiuscola nel caso dei nomi di popoli e degli aggettivi qualificativi etnici (risvegliare in ogni Americano il sentimento dell'unità, mafia Russa), causa l'interferenza non solo dallo sloveno ma

21 Viste da una prospettiva esogena, le regole che governano l'uso della virgola in sloveno sono rigide e fisse, spesso del tutto diverse da quanto prevedono le altre lingue nei propri sistemi. Benché non esista il riferimento a una regola ortografica concreta, nel sistema scolastico sloveno ai bambini viene insegnato un gioco di parole (filastrocca) con cui si cerca di imporre l'automatizzazione dell'impiego della virgola davanti ai connettivi più frequenti (tra cui appunto le congiunzioni completiva, temporale, causale e condizionale e i pronomi relativi): »Pred ki, ko, ker, da, če vejica skače« ( $L a$ virgola spunta davanti a che, quando, perché e se.). La conseguenza di quest'automatizzazione è che la virgola negli scritti dei discenti slovenofoni in L2 trova posto in situazioni analoghe senza motivi e giustificazioni riconducibili alle regole della lingua target. 
anche dall'inglese in quanto in entrambe le lingue la maiuscola è obbligatoria sia con i nomi dei popoli che con la forma aggettivale; ${ }^{22}$

- casi in cui nonostante la natura dell'errore sia ortografica, l'errore a monte è di tipo fonologico (causa la non corrispondenza tra la pronuncia e la grafia dei due sistemi):

$\begin{array}{ccc}\text { pronuncia } & \begin{array}{c}\text { grafema } \\ \text { sloveno }\end{array} & \text { grafema } \\ \text { italiano }\end{array}$

(58) Per primo il denaro

/ts/

c

viene da un crimine, poi lo

piacciano nelle banche e alla

fine viene investito.

(59) Questi servizi mafiozi

$|\mathrm{z}|$

Z

S

assumano una gran varietà di

forme.

(60) $\operatorname{droge}$ (f., plur)

$/ \mathrm{g} /$

gh

(61) bance (f., plur)

/k/

$\begin{array}{ll}\mathrm{g} & \mathrm{gh} \\ \mathrm{k} & \mathrm{ch}\end{array}$

\section{FALSI AMICI}

Le interferenze attribuibili al settore dei «falsi amici» presentano errori di natura ortografica, morfologico-sintattica, derivazionale e lessicale.

Errori ortografici - casi in cui le parole condividono la stessa origine etimologica, ma hanno subìto evoluzioni differenti:
sloveno
origine etimologica comune
(62) la mafia manipula droge manipulirati
lat. manipŭlus

${ }^{22}$ Per quanto riguarda l'italiano, sul sito dell'Accademia della Crusca Luca Serianni precisa: «Per i nomi di popoli (o etnici) bisogna distinguere: la minuscola è oggi obbligatoria se si tratta di aggettivi («le strade francesi»), decisamente preferita in riferimento a un singolo individuo («il greco aveva conservato fino allora un silenzio pregnante» P. Levi), mentre si alterna con la maiuscola nel plurale («e sì che i tedeschi non li batte nessuno in nulla» Fenoglio / «i Tedeschi lo avevano preso in seguito alla spiata di un qualche delatore» Morante)» (Serianni \& Nencioni, 1991). 
Errori morfosintattici - il transfer negativo si manifesta nel trasferimento della marca del genere dei nomi corrispondenti dallo sloveno e nel conseguente impatto anche sugli accordi necessari:

sloveno

(63) la sua potere

piccoli associazioni

maggiori risorsi nazionali

(64) La discrezione è il parte più importante dell'attività mafiosa.

(65) (...) che il problema del traffico a Roma diventi una dei temi della campagna elettorale moč (f. sing.)/potere

združenje (m. sing.)/associazione

$\operatorname{vir}$ (m. sing.)/risorsa; sredstvo (n. sing.)/ risorsa

$\operatorname{del}(\mathrm{m}$. sing.)/parte

tema (f. sing.)/tema, argomento

L'interferenza si rileva anche in alcuni procedimenti sintattici. Si segnala in primis il caso di errata concordanza a senso in cui il predicato viene realizzato con la prima persona plurale mentre il soggetto è espresso con un sintagma nominale alla terza persona plurale. Quest'interferenza è dovuta al fatto che in situazioni analoghe (cioè quando il parlante si considera incluso nel gruppo espresso dal S) in sloveno si preferisce la concordanza a senso e non la concordanza grammaticale, come in italiano. Segue un esempio illustrativo:

\begin{tabular}{l|l} 
& sloveno \\
\hline $\begin{array}{c}\text { (66) Persone comuni corriamo } \\
\text { il rischiono di venire in contatto con } \\
\text { il denaro sporco. }\end{array}$ & Navadni ljudje tvegamo... \\
\end{tabular}

${ }^{23}$ Navadni ljudje/Persone comuni; tvegamo/rischiamo (voce del verbo tvegati/rischiare, I pers. plur.) 
Sono imputabili all'influsso dello sloveno anche alcuni errori nella distribuzione dei costituenti della frase italiana. Questi vanno attribuiti da una parte alla rigidità del sistema sloveno, ad esempio per quanto riguarda la posizione dell'aggettivo, che in sloveno è solo prenominale e non rappresenta un fattore determinante per la distinzione tra la funzione descrittiva e quella restrittiva (ess. 67-68), e dall'altra invece alla sua flessibilità: si pensa soprattutto alla flessibilità della posizione dell'oggetto diretto (es. 69) all'interno della frase (e soprattutto rispetto al predicato), all'inesistenza della ripresa dell'oggetto diretto con il clitico in caso di dislocazione (ess. 70-71) e alla possibilità di introdurre un numero non limitato di elementi tra l'ausiliare e il participio passato di una forma verbale composta (ess. 72-74).

(67) (...) neanche nei più sicuri affari non è sempre come dovrebbe essere.

(68) per costruire i fondamenti della sua, più di cento anni vecchia, organizzazione criminale

(69) Con la proibizione non si risolve niente, questo abbiamo già visto cento anni fa.

(70) Un certo controllo sulla mafia $\varnothing$ esercitava «Cupola».

(71) Questo cambiamento del dominio $\varnothing$ notava anche il magistrato Giovanni Falcone.

(72) (...) perché hanno senza la minima creatività copiato uno spot pubblicitario americano.

(73) Quando è stato chiesto ai candidati cosa ne pensassero, hanno senza girarci intorno, tutti risposto di sì.

(74) L'opinione pubblica sulla immagine parastatale della mafia si era per vari motivi tanto legislativi come gerarchici rivelata falsa.

Errori di morfologia derivazionale - casi di applicazioni di suffissi indotti da alcune corrispondenze tra le due lingue. L'esempio (75) contiene l'illustrazione di un caso in cui si tratta della traduzione dell'espressione slovena combinata con l'applicazione del processo di derivazione (suffisso $\mathrm{V}>\mathrm{N}$ ) per esprimere l'azione e il risultato che ne consegue (sul modello: pagare $>$ pagamento; giacere $>$ giacimento): 


\begin{tabular}{l|l} 
& sloveno \\
\hline $\begin{array}{c}\text { (75) senza qualsiasi pensamento (pensare } \\
>\text { pensamento) }\end{array}$ & brez kakršnegakoli razmišljanja
\end{tabular}

Errori lessicali - il seguente tipo di interferenza riguarda un uso sbagliato di espressioni o locuzioni a causa della vicinanza formale e/o semantica di uno dei loro componenti:

\begin{tabular}{c|l} 
& \multicolumn{1}{|c}{ sloveno } \\
\hline $\begin{array}{c}\text { (76) Ognuno di noi, senza saperlo, può entrare } \\
\text { nel supermercato dove si opera con i soldi } \\
\text { sporchi. Così tutti abbiamo da fare con } \\
\text { il denaro invisibile... }\end{array}$ & $\begin{array}{l}\text { avere da fare/imeti opravke, imeti nekaj } \\
\text { za delati } \\
\text { avere a che fare con/imeti opraviti z }\end{array}$
\end{tabular}

\begin{tabular}{l|l} 
& \multicolumn{1}{|c}{ sloveno } \\
\hline $\begin{array}{c}\text { (77) A questo punto la domanda che si apre } \\
\begin{array}{c}\text { non riguarda alla realizzazione del } \\
\text { detto... }\end{array}\end{array}$ & $\begin{array}{l}\text { riguardare qualcosa/nanašati se na } \\
(>* \text { riguardare } a)\end{array}$
\end{tabular}

\section{CONCLUSIONI}

La natura delle prove scritte prese in considerazione per questo studio ha prodotto lavori individuali in cui gli autori hanno dovuto dare il meglio di sé per sintetizzare in forma ridotta testi di partenza per loro sconosciuti. Di conseguenza gli scritti hanno dato la possibilità di individuare le zone più insidiose, al contempo comuni e ripetitive, da cui è stato possibile enucleare i procedimenti cognitivi legati all'influsso della lingua materna a monte degli errori di produzione nella lingua straniera. La preparazione linguistica degli apprendenti è fondata sia sull'apprendimento naturale (sebbene di natura individuale e di dimensioni e intensità del tutto differenti da soggetto a soggetto) sia sull'apprendimento guidato incentrato sullo studio dettagliato della norma linguistica italiana. Questi presupposti hanno fatto sì che alcune tipologie di errori siano quasi del tutto 
assenti nel corpus analizzato, sebbene dall'analisi risulti che esiste tutta una serie di situazioni critiche sulle quali è necessario ancora lavorare e esercitarsi. Moltissimo è stato fatto sul campo degli studi contrastivi per individuare le ragioni dell'interferenza negativa dallo sloveno (basti pensare al considerevole e notevole lavoro di Miklič, 1981, 1983, 1992, per quanto riguarda l'uso delle forme verbali in italiano), eppure i curricula sembrano spesso ignorare o perlomeno sottovalutare la necessità di riferimento sistematico ai dettagli formali dell'input e di uno studio più accurato delle situazioni difficili per i parlanti slovenofoni, soprattutto durante i percorsi scolastici del primo ciclo di istruzione. I risultati di quest'analisi non fanno altro che ribadire la necessità dell'inserimento di questi contenuti nei processi di apprendimento.

I recenti studi sull'acquisizione di lingue straniere per fortuna hanno portato al superamento delle teorie krasheniane sulla sufficienza dell'input comprensibile o quella dell'insegnamento comunicativo, per dare luogo a una cosciente integrazione dei diversi fattori che provvedono un apporto individuale e collettivo all'apprendimento. L'insegnamento diretto, la consapevolezza dei meccanismi linguistici (noticing) e l'attenzione alla forma (focus on form) aiutano notevolmente gli apprendenti a mettere ordine nel proprio input nonché a facilitare la comprensione e a stimolare o sostenere i processi naturali di acquisizione (cf. Schmidt, 1995; Grassi, 2015).

In questo quadro si colloca anche la trattazione dell'errore che, per mantenere un focus on form comunque fedele e coerente ai precetti comunicativi, esige un feedback correttivo e riparativo - prassi adottata in forma attenta e meticolosa anche durante il corso in cui è stata svolta l'attività considerata e studiata per questo lavoro. È nostro augurio pertanto che quanto sostiene Grassi possa veramente consolidarsi come parte imprescindibile dell'insegnamento guidato nel processo di apprendimento di lingue straniere:

una didattica realmente acquisizionale dovrebbe [...] costruire la correzione, e quindi l'evoluzione della competenza, a partire dalle 'regole' sottostanti alle produzioni interlinguistiche divergenti dal target, che andrebbero pertanto fatte emergere in forma conscia e verbalizzata, così da poter essere 
scardinate, scomposte e ricomposte in regole più targetlike. (Grassi, 2015, p. 186)

\section{BIBLIOGRAFIA}

Ahlin, M., Bokal, L., Gložančev, A., Hajnšek-Holz, M., Humar, M., Keber, J. ... Vojnovič, N. (1994). Slovar slovenskega knjižnega jezika. Ljubljana: SAZU/DZS.

Andorno, C. (2012). Varietà di esiti dell'apprendimento dell'italiano nella varietà dei contesti di apprendimento: possibilità e limiti dell'acquisizione naturale. In R. Grassi (Ed.), Nuovi contesti d'acquisizione e insegnamento: l'italiano nelle realtà plurilingui (pp. 157-173). Perugia: Guerra.

Andorno, C. (2015). Errori, regole ed eccezioni nell'apprendimento. In N. Grandi (Ed.), (pp. 161-176).

Berruto, G. (2015). Le regole in linguistica. In N. Grandi (Ed.), (pp. 43-61).

Bertuccelli Papi, M. (2001). Frasi subordinate al participio: Participio passato. In L. Renzi, G. Salvi \& A. Cardinaletti (Eds.), Grande grammatica italiana di consultazione (Vol. II, pp. 593-604). Bologna: il Mulino.

Benincà, P., \& Cinque, G. (2001). Frasi subordinate al participio: Participio presente. In L. Renzi, G. Salvi \& A. Cardinaletti (Eds.), Grande grammatica italiana di consultazione (Vol. II, pp. 604-609). Bologna: il Mulino.

Bley-Vroman, R. (1983). The comparative fallacy in interlanguage studies: the case of sistematicity. Language learning, 33, 1-17.

Cacchione, A. (2011). L'italiano scritto degli studenti universitari italiani/italofoni. Cuadernos de Filologia Italiana, vol.18, 11-30.

Cadorna, G.R. (1988). Dizionario di Linguistica. Roma: Armando.

Corder, S.P. (1971). Idiosyncratic dialects and error analysis. Interantional Review of Applied Linguistics, Vol. 9, No. 2, 147-160.

Donati, C. (2013). L'acquisizione del linguaggio. In G. Graffi \& S. Scalise (Eds.), Le lingue e il linguaggio (pp. 277-299). Bologna: Il Mulino.

Fornasiero, S., \& Tamiozzo Goldman, S. (2013). Scrivere l'italiano. Galateo della comunicazione scritta. Bologna: Il Mulino.

Grandi, N. (Ed.). (2015). La grammatica e l'errore. Le lingue naturali tra regole, loro violazioni ed eccezioni. Bologna: Bononia University Press.

Grassi, R. (2015). Reazioni all'errore ed eccezioni all'inevitabilità delle regole nella Didattica Delle Lingue Seconde. In N. Grandi (Ed.), (pp. 177-191). 
Jesenšek, M. (1998). Deležniki in deležja na -č in -ši: razširjenost oblikv slovenskem knjižnem jeziku 19. stoletja. Maribor: Slavistično društvo.

James, C. (1998). Errors in Language Learning and Use. London and New York: Longman.

Lennon, P. (1991). Error: Some Problems of Definition, Identification, and Distinction. Applied Lingustics, 12 (2), 180-195.

Logar, N., Arhar Holdt, Š., \& Erjavec T. (2016). Slovenski strokovni jezik: Korpusni opis trpnika. In E. Kržišnik \& M. Hladnik (Eds.), OBDOBJA 35 Toporišičeva Obdobja (pp. 237-245). Ljubljana: Znanstvena založba Filozofske fakultete.

Lonzi, L. (1988). Frasi subordinate al gerundio. In L. Renzi, G. Salvi \& A. Cardinaletti (Eds.), Grande grammatica italiana di consultazione (Vol. II, pp. 571-592). Bologna: il Mulino.

Miklič, T. (1981). Kriteriji izbire med perfektom in imperfektom v primerjavi s kriteriji izbire med dovršniki in nedovršniki. Ljubljana: Filozofska fakulteta Univerze Edvarda Kardelja, PZE za romanske jezike in književnosti.

Miklič, T. (1983). L'opposizione italiana PERFETTO vs IMPERFETTO e l'opposizione slovena DOVRŠNOST vs NEDOVRŠNOST nella verbalizzazione delle azioni passate. Linguistica, 23, 53-123.

Miklič, T. (1992). La Consecutio Temporum in sloveno e in italiano: alcune osservazioni. In A.G. Mocciaro \& G. Soravia (Eds.), L'Europa linguistica: contatti, contrasti, affinità di lingue: atti del XXI Congresso Internazionale [della Società linguistica italiana], Catania, 10-12 settembre 1987 (pp. 189-218). Roma: Bulzoni.

Mikolič Južnič, T. (2017). Kontrastivni vidiki prevajanja italijanskih neosebnih glagolskih oblik $\mathrm{v}$ slovenščino: korpusna analiza gerundija. In A. Pisanski Peterlin \& M. Schlamberger Brezar (Eds.), Prevodoslovno usmerjene kontrastivne študije (pp. 32-49). Ljubljana: Znanstvena založba Filozofske fakultete.

Renzi, L. (1988). L'articolo. In L. Renzi, G. Salvi \& A. Cardinaletti (Eds.), Grande grammatica italiana di consultazione (Vol. I, pp. 357-424). Bologna: il Mulino.

Selinker, L. (1972). Interlanguage. International Review of Applied Linguistics, Vol. 10 No. 3, 209-231.

Serianni, L., \& Nencioni, G. (1991). Risposta al quesito della Scuola media "Leon Battista Alberti" di Mantova sull'uso della maiuscola nei nomi di popolo. Crusca per Voi, ${ }^{\circ}$ 2, aprile 1991, 10-11, Retrieved from http:// 
www.accademiadellacrusca.it/it/lingua-italiana/consulenza-linguistica/domande-risposte/uso-maiuscole-minuscole.

Schmidt, R. (1995). Consciousness and foreign language learning: A tutorial on the role of attention and awareness in learning. In R. Schmidt (Ed.), Attention and awareness in foreign language learning (pp. 1-63). Honolulu: Hawai'i: University of Hawai'i, Second Language Teaching \& Curriculum Center.

Toporišič, J. (2004). Slovenska slovnica. Maribor: Obzorja.

Vanelli, L. (1979). Una forma suppletiva dell'articolo e la sua fonosintassi. Rivista di grammatica generativa, 4, 1-2, 165-181.

Visigalli, M. (2015). La percezione e la correzione dell'errore in italiano L2: 1'influenza della L1 sulla riparazione spontanea di errori pragmatici. In M. Chini (Ed.), Il Parlato in [italiano] L2: aspetti pragmatici e prosodici (pp. 221-240). Milano: Franco Angeli.

Weinreich, U. (1974). Lingue in contatto. Torino: Boringhieri. ZRC SAZU (Eds.). (2001). Slovenski pravopis. Ljubljana: ZRC SAZU.

\section{Corpus}

Cosenza, G. (2012, Jan 25), Se la pubblicità fa leva sull'orgoglio patrio. Il Fatto Quotidiano. Retrieved from https://www.ilfattoquotidiano.it/2012/01/25/ pubblicita-leva-sull\%E2\%80\%99orgoglio-patrio/186387.

Ginsborg, P. (1998). La natura della mafia. In P. Ginsborg, L'Italia del tempo presente (pp. 365-368). Torino: Einaudi.

Smith, D.M. (2011). Storia d'Italia dal 1861 al 1997 (pp. 460-465). Roma-Bari: Laterza.

Fazio, F. intervista Pietro Grasso (2011, Dec 12) RAITRE, Che tempo che fa.

Riassunto: Questo contributo mira ad affrontare e indicare i punti nevralgici dell'interferenza della lingua madre negli scritti degli apprendenti slovenofoni in lingua italiana. La questione viene presentata a partire dal confronto tra le norme linguistiche in sloveno e italiano in relazione alle difficoltà riscontrate in contesti concreti. L'obiettivo del contributo è individuare le ipotesi che stanno alla base delle scelte linguistiche degli apprendenti. Sono illustrati gli errori commessi nella scelta dei paradigmi verbali (consecutio temporum, dubbi di scelta tra i perfetti e l'imperfetto e tra le forme esplicite e implicite, alcuni costrutti passivi, il congiuntivo) nonché le difficoltà d'uso dell'articolo e alcuni aggettivi e pronomi (possessivi, dimostrativi e relativi). Sono inoltre documentati e commentati gli esempi di errori nell'uso della virgola e casi di interferenze attribuibili al settore dei «falsi amici» (errori di natura ortografica, morfologico-sintattica, derivazionale e lessicale). 
I risultati di quest'analisi ribadiscono la necessità di una integrazione cosciente dell'insegnamento diretto, della consapevolezza dei meccanismi linguistici (noticing) e dell'attenzione alla forma (focus on form) nei processi di apprendimento per mettere ordine nell'input degli apprendenti e per facilitare la comprensione e per stimolare i processi naturali di acquisizione.

Parole chiave: apprendenti slovenofoni di italiano L2, analisi contrastiva, interferenza della lingua madre, riassunto, analisi degli errori 